\title{
Beyond the Gross-Pitaevskii approximation: Local density versus correlated basis approach for trapped bosons
}

\author{
A. Fabrocini ${ }^{1}$ and A. Polls ${ }^{2}$ \\ ${ }^{1}$ Dipartimento di Fisica, Università di Pisa, I-56100 Pisa, Italy \\ ${ }^{2}$ Departament d'Estructura i Constituents de la Matèria, Universitat de Barcelona, E-08028 Barcelona, Spain \\ (Received 28 January 1999)
}

\begin{abstract}
We study the ground state of a system of Bose hard spheres trapped in an isotropic harmonic potential to investigate the effect of the interatomic correlations and the accuracy of the Gross-Pitaevskii equation. We compare a local-density approximation, based on the energy functional derived from the low-density expansion of the energy of the uniform hard-sphere gas, and a correlated wave-function approach, which explicitly introduces the correlations induced by the potential. Both higher-order terms in the low-density expansion, beyond Gross-Pitaevskii, and explicit dynamical correlations have effects of the order of percent when the number of trapped particles becomes similar to that attained in recent experiments $\left(N \sim 10^{7}\right)$.
\end{abstract}

[S1050-2947(99)00109-2]

PACS number(s): 03.75.Fi, 05.30.Jp, 32.80.Pj

The recent discovery of Bose-Einstein condensation of magnetically trapped alkali atoms has generated a huge amount of theoretical investigations. A review of the present situation can be found in Refs. [1,2]. Present experimental conditions are such that the atomic gas is very dilute, i.e., the average distance among the atoms is much larger than the range of the interaction. As a consequence, the physics should be dominated by two-body collisions, generally well described in terms of the $s$-wave scattering length. The case of a positive scattering length is equivalent to consider a very dilute system of hard spheres, whose diameter coincides with the scattering length itself. So, the Gross-Pitaevskii (GP) theory for weakly interacting bosons seems the logical tool to study these systems and most of the present days theoretical work is founded on (or has its starting point in) this theory [3]. However, in very recent experiments the number of trapped atoms has spectacularly increased reaching $N$ values of the order $10^{6}$ and $10^{7}$ atoms [4]. Therefore, it seems logical to ask for a deeper study of the effect of the interatomic correlations and of the accuracy of the GrossPitaevskii scheme in this new scenario. First-order corrections to the mean field have been evaluated in Refs. [1,5]. The dense condensate case was studied in a slave boson representation in Ref. [6]. Exact quantum Monte Carlo methods $[7,8]$ have been also employed, but only up to $N=10^{5}$.

Here we study the ground state of a system of Bose hard spheres trapped by a harmonic potential. More precisely, we consider hard spheres with a diameter of $52.9 \AA$, corresponding to the $s$-wave triplet-spin scattering length of ${ }^{87} \mathrm{Rb}$, in an isotropic harmonic trap characterized by an angular frequency $\omega / 2 \pi=77.78 \mathrm{~Hz}$. We also examine the large $N$ atomic sodium case of Ref. [4]. We use and compare two methods: (i) a local-density approximation (LDA) based on an energy functional derived by the low-density expansion of the energy of an uniform hard-sphere gas and (ii) a correlated basis function (CBF) approach, which explicitly takes into account the dynamical correlations induced by the potential and which is not, in principle, limited to purely repulsive interactions.
LDA theory. In the case of a uniform hard-spheres Bose gas, a perturbation theory in the expansion parameter $x$ $=n a^{3}$, where $n$ is the density of the system, leads to the following low-density expansion for the energy density [9]:

$$
\begin{aligned}
\frac{E}{V}= & \frac{2 \pi n^{2} a \hbar^{2}}{m}\left[1+\frac{128}{15}\left(\frac{n a^{3}}{\pi}\right)^{1 / 2}\right. \\
& \left.+8\left(\frac{4}{3} \pi-\sqrt{3}\right)\left(n a^{3}\right) \ln \left(n a^{3}\right)+O\left(n a^{3}\right)\right] .
\end{aligned}
$$

The energy functional associated with the GrossPitaevskii theory is simply obtained in the local-density approximation by keeping only the first term in expansion (1):

$$
E_{G P}[\psi]=\int d \mathbf{r}\left[\frac{\hbar^{2}}{2 m}|\nabla \psi(r)|^{2}+\frac{m}{2} \omega^{2} r^{2}|\psi|^{2}+\frac{2 \pi \hbar^{2} a}{m}|\psi|^{4}\right],
$$

where the wave-function $\psi$ is normalized to the total number of atoms.

By performing a functional variation of $E_{G P}[\psi]$ one finds the Euler-Lagrange equation, known as the Gross-Pitaevskii equation,

$$
\left[-\frac{\hbar^{2}}{2 m} \nabla^{2}+\frac{m}{2} \omega^{2} r^{2}+\frac{4 \pi \hbar^{2} a}{m}|\psi|^{2}\right] \psi=\mu \psi,
$$

where $\mu$ is the chemical potential. This equation has the form of a nonlinear stationary Schrödinger equation, and it has been solved for several types of traps using different numerical methods [10-13].

A logical step further, in the spirit of LDA, is to include into the energy functional the next terms of correlation energy (1). It is convenient to simplify the notation by expressing lengths and energies in harmonic oscillator units. The spatial coordinates, the energy, and the wave function are 
rescaled as $\mathbf{r}=a_{H O} \overline{\mathbf{r}}, E=\hbar \omega E_{1}, \quad$ and $\quad \psi(r)$ $=\left(N / a_{H O}^{3}\right)^{1 / 2} \psi_{1}(\bar{r})$, where $a_{H O}=(\hbar / m \omega)^{1 / 2}$ and $\psi_{1}(\bar{r})$ is normalized to unity.

By taking into account the next terms of the correlation energy, we obtain a modified Gross-Pitaevskii (MGP) energy functional,

$$
\begin{aligned}
E_{M G P}\left[\psi_{1}\right]= & \int d \overline{\mathbf{r}}\left\{\frac{1}{2}\left|\nabla_{\bar{r}} \psi_{1}(\bar{r})\right|^{2}+\frac{1}{2} \bar{r}^{2}\left|\psi_{1}(\bar{r})\right|^{2}\right. \\
& +2 \pi \bar{a} N\left|\Psi_{1}(\bar{r})\right|^{4}\left[1+\frac{1}{\pi} \frac{128}{15}\left[N \bar{a}^{3}\left|\psi_{1}(\bar{r})\right|^{2}\right]^{1 / 2}\right. \\
& +8\left(\frac{4 \pi}{3}-\sqrt{3}\right) N \bar{a}^{3}\left|\psi_{1}(\bar{r})\right|^{2} \\
& \left.\left.\times \ln \left[N \bar{a}^{3}\left|\psi_{1}(\bar{r})\right|^{2}\right]\right]\right\},
\end{aligned}
$$

and a corresponding modified Gross-Pitaevskii equation,

$$
\begin{aligned}
& {\left[-\frac{1}{2} \nabla_{\frac{r}{r}}^{2}+\frac{1}{2} \bar{r}^{2}+4 \pi \bar{a} N\left|\psi_{1}(\bar{r})\right|^{2}+5 \pi^{1 / 2} \bar{a}^{5 / 2} N^{3 / 2} \frac{128}{15}\left|\psi_{1}(\bar{r})\right|^{3}\right.} \\
& \quad+8 \pi \bar{a}^{4} N^{2}\left(\frac{4 \pi}{3}-\sqrt{3}\right)\left|\psi_{1}(\bar{r})\right|^{4} \\
& \left.\quad \times\left\{6 \ln \left[N \bar{a}^{3}\left|\psi_{1}(\bar{r})\right|^{2}\right]+2\right\}\right] \psi_{1}(\bar{r}) \\
& =\mu_{1} \psi_{1}(\bar{r}),
\end{aligned}
$$

where $\bar{a}=a / a_{\mathrm{HO}}$ and $\mu_{1}$ is the chemical potential in harmonic oscillator units. Equation (5) contains extra nonlinear terms in $\psi_{1}$.

CBF approach. Correlated basis function theory is a powerful tool to study strongly interacting many-body systems (for a review, see Ref. [14]). In particular, it was used to study the hard-spheres homogeneous Fermi gas in order to ascertain the accuracy of low-density-type expansions [15].

For $N$ interacting bosons, at $T=0$ temperature, described by the Hamiltonian

$$
H=-\frac{\hbar^{2}}{2 m} \sum_{i} \nabla_{i}^{2}+\sum_{i} V_{\text {ext }}\left(\mathbf{r}_{i}\right)+\sum_{i<j} V\left(r_{i j}\right),
$$

where $V_{\text {ext }}\left(\mathbf{r}_{i}\right)$ is the confining potential and $V\left(r_{i j}\right)$ is the interatomic potential, the $\mathrm{CBF}$ ground-state wave function is

$$
\psi_{C B F}(1, \ldots, N)=F(1, \ldots, N) \psi_{M F}(1, \ldots, N),
$$

where $F(1, \ldots, N)$ is a many-body correlation operator applied to the mean-field wave-function $\psi_{M F}$. The advantage of using a correlated basis lies in the fact that nonperturbative effects, as the short-range repulsion for the hard spheres, may be directly inserted into the correlation.

The simplest correlation operator has the Jastrow form [16],

$$
F(1, \ldots, N)=\prod_{i<j} f_{J}\left(r_{i j}\right)
$$

where the Jastrow correlation function, $f_{J}(r)$, depends on the interparticle distance only. $f_{J}(r)$ is variationally determined by minimizing the expectation value of $E_{C B F}$ $=\left\langle\psi_{C B F}|H| \psi_{C B F}\right\rangle /\left\langle\psi_{C B F} \mid \psi_{C B F}\right\rangle . \quad E_{C B F}$ may be evaluated either by Monte Carlo techniques or by cluster expansions of the needed one- and two-body densities, $\rho_{1}\left(\mathbf{r}_{1}\right)$ and $\rho_{2}\left(\mathbf{r}_{1}, \mathbf{r}_{2}\right)$.

The energy per particle can be written as $E_{C B F} / N=T_{\rho}$ $+V_{e}+V_{\text {corr }}$, where

$$
\begin{gathered}
T_{\rho}=\frac{1}{N} \frac{\hbar^{2}}{2 m} \int d \mathbf{r}_{1}\left[\vec{\nabla}_{1} \rho_{1}^{1 / 2}\left(\mathbf{r}_{1}\right)\right]^{2}, \\
V_{e}=\frac{1}{N} \int d \mathbf{r}_{1} \rho_{1}\left(\mathbf{r}_{1}\right) V_{\text {ext }}\left(\mathbf{r}_{1}\right),
\end{gathered}
$$

and the correlation energy, $V_{\text {corr }}$, is

$$
\begin{aligned}
V_{c o r r}= & \frac{1}{N} \frac{1}{2} \int d \mathbf{r}_{1} \int d \mathbf{r}_{2} \rho_{2}\left(\mathbf{r}_{1}, \mathbf{r}_{2}\right)\left[V\left(r_{12}\right)\right. \\
& \left.-\frac{\hbar^{2}}{2 m} \vec{\nabla}^{2} \ln f_{J}\left(r_{12}\right)\right]-\frac{1}{N} \frac{\hbar^{2}}{2 m} \int d \mathbf{r}_{1} \int d \mathbf{r}_{2} \rho_{2}\left(\mathbf{r}_{1}, \mathbf{r}_{2}\right) \\
& \times \vec{\nabla} \ln f_{J}\left(r_{12}\right) \cdot \vec{\nabla} \ln \rho_{1}^{1 / 2}\left(\mathbf{r}_{1}\right)
\end{aligned}
$$

where $\rho_{1}$ is normalized to $N$.

$\rho_{2}$ may be calculated by cluster expansion in powers of the dynamical correlation, $h(r)=f_{J}^{2}(r)-1$, and the integral hypernetted-chain (HNC) equations [17] may be used to sum infinite classes of cluster diagrams.

Given the low density of the trapped bosons' system, it looks plausible to start from a lowest-order cluster expansion. In this approximation $\rho_{2}^{(L O)}\left(\mathbf{r}_{1}, \mathbf{r}_{2}\right)$ $=\rho_{1}\left(\mathbf{r}_{1}\right) \rho_{1}\left(\mathbf{r}_{2}\right) f_{J}^{2}\left(r_{12}\right)$ and

$$
V_{\text {corr }}^{(L O)}=\frac{1}{N} \frac{1}{2} \int d \mathbf{r}_{1} \int d \mathbf{r}_{2} \rho_{2}^{(L O)}\left(\mathbf{r}_{1}, \mathbf{r}_{2}\right) V_{J F}\left(r_{12}\right)
$$

where $V_{J F}(r)=V(r)+\left(\hbar^{2} / m\right)\left[\vec{\nabla} \ln f_{J}(r)\right]^{2}$ is the JacksonFeenberg potential.

The minimization of $E_{C B F}^{(L O)}$ with respect to $\rho_{1}$ leads to the lowest-order correlated Hartree $\left(\mathrm{CH}_{L O}\right)$ equation,

$$
\begin{aligned}
& {\left[-\frac{\hbar^{2}}{2 m} \nabla^{2}+V_{\text {ext }}(\mathbf{r})+\int d \mathbf{r}_{1} \rho_{1}\left(\mathbf{r}_{1}\right) f_{J}^{2}(s) V_{J F}(s)\right] \rho_{1}^{1 / 2}(\mathbf{r})} \\
& \quad=\mu \rho_{1}^{1 / 2}(\mathbf{r})
\end{aligned}
$$

with $s=\left|\mathbf{r}-\mathbf{r}_{1}\right|$.

The optimal choice for the Jastrow factor would be the one satisfying the Euler equation $\delta E_{C B F} / \delta f_{J}(r)=0$. Otherwise, parametrized functional forms may be chosen whose parameters are found through the minimization process. We adopt here the correlation function minimizing the lowestorder energy of a homogeneous Bose gas with a healing condition at a distance $d$ (taken as a variational parameter). For the hard-spheres case, $f_{J}(r<a)=0$ and $f_{J}(r>a)$ $=u(r) / r$, where $u(r)$ is solution of the Schrödinger-like equation $-u^{\prime \prime}=K^{2} u . f_{J}(r)$ has the form [18] 


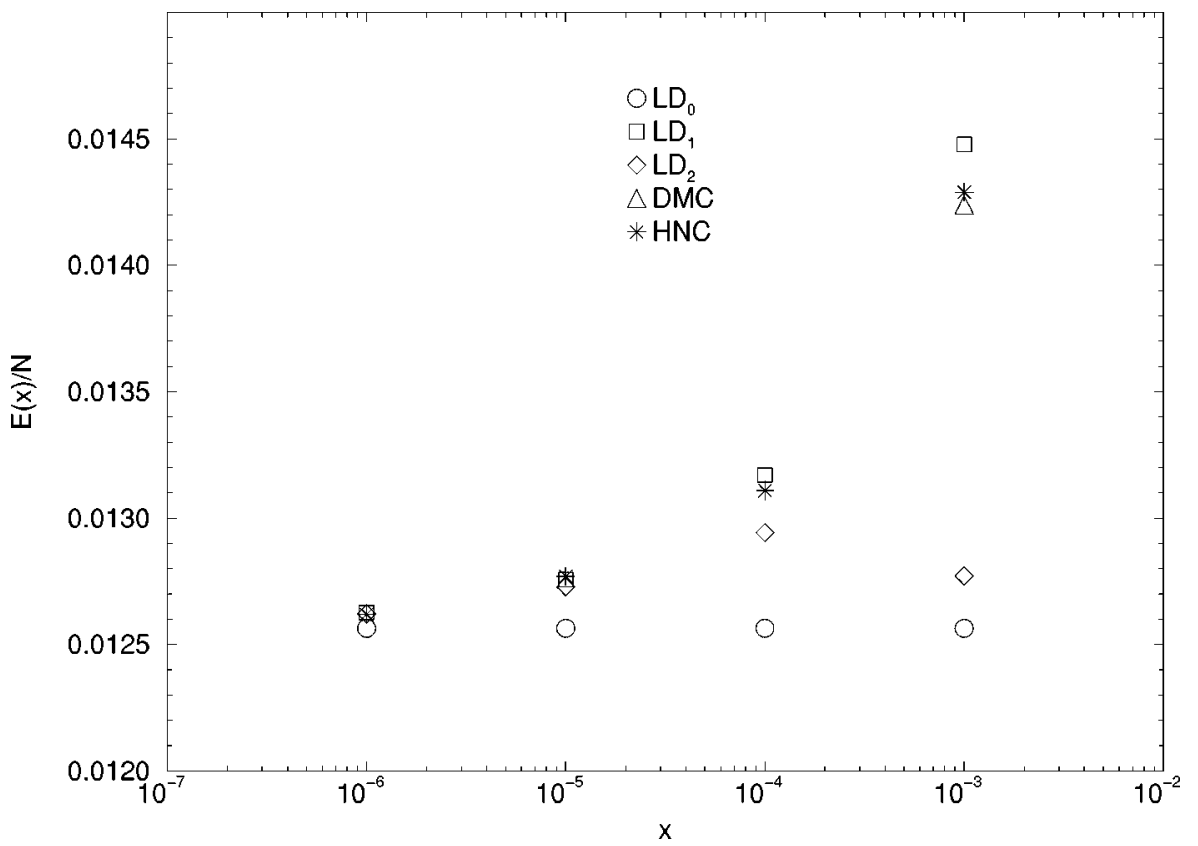

FIG. 1. Energy per particle (in units of $\hbar^{2} / 2 m a^{2}$ ) for homogeneous hard spheres in function of $x$. The symbols correspond to the low-density expansion results [Eq. (1)] obtained by keeping only the first term $\left(\mathrm{LD}_{0}\right)$ or by adding the second $\left(\mathrm{LD}_{1}\right)$ and the third $\left(\mathrm{LD}_{2}\right)$ ones, and to the diffusion Monte Carlo (DMC) and HNC energies.

$$
f_{J}(r)=\frac{d}{r} \frac{\sin [K(r-a)]}{\sin [K(d-a)]},
$$

where the healing conditions, $f_{J}(r \geqslant d)=1$ and $f_{J}^{\prime}(r=d)$ $=0$, are fixed by the relation: $\cot [K(d-a)]=(K d)^{-1}$.

Results. We begin by briefly discussing the boson hardspheres homogeneous case. It was shown in Ref. [18] that the lowest-order cluster energy of an infinite system of bosonic hard spheres, correlated by the Jastrow factor (14), asymptotically tends to the first term of low-density expansion (1) when the healing distance goes to infinity. We have numerically checked this behavior. The situation changes if all the many-body cluster terms are included via HNC summation, since the computed energy shows a clear minimum in $d$. Some results are given in Fig. 1 for various $x$-values. The energies have been multiplied by $10^{3(2,1)}$ at $x$ $=10^{-6(-5,-4)}$, respectively. The figure shows the energy estimates computed by retaining different expansion terms [the local-density $\left(\mathrm{LD}_{0}\right)$ values correspond to the first term in Eq. (1), whereas the $\mathrm{LD}_{1}$ and $\mathrm{LD}_{2}$ ones are obtained by adding the second and third terms, respectively], the HNC energies and, at the highest $x$ value, the exact energy, evaluated by a diffusion Monte Carlo method [19]. The quality of the HNC results is excellent as they practically coincide with the exact ones at all the densities (the low density value at low $x$ and the Monte Carlo one at large $x$ ). The convergence of expansion (1) at large $x$ appears to be rather poor, pointing to the relevance of successive contributions.

The Gross-Pitaevskii, the modified Gross-Pitaevskii, and the lowest-order correlated Hartree equations for $\psi_{1}$ have been solved by the steepest descent method [20] for the isotropic harmonic-oscillator trap previously described. An initial trial state is projected onto the minimum of the functional by propagating it in imaginary time. In practice, one chooses an arbitrary time step $\Delta t$ and iterates the equation

$$
\psi_{1}\left(\mathbf{r}_{1}, t+\Delta t\right) \approx \psi_{1}\left(\mathbf{r}_{1}, t\right)-\Delta t H \psi_{1}\left(\mathbf{r}_{1}, t\right)
$$

by normalizing $\psi_{1}$ to 1 at each iteration. When the number of particles becomes very large the time step, which governs the rate of convergence, should be taken accordingly small. The number of iterations substantially increases and it is convenient to start the convergence process from a reasonable wave function [for the GP and MGP equations we start from the ground-state harmonic-oscillator function, which minimizes energy functional (2), while, in the $\mathrm{CH}_{L O}$ case, we start from the GP solution].

We find that the lowest-order approximation in the finite system shows a behavior similar to the infinite case. In particular, the solution of Eq. (13) provides an energy (in reduced unities) very close to the Gross-Pitaevskii one when the healing distance becomes very large. In fact, for $N$ $=10^{5}$ and $\bar{a}=4.33 \times 10^{-3}$, corresponding to the ${ }^{87} \mathrm{Rb}$ scattering length, we obtain $E_{1}^{(L O)} / N=12.57,12.28$, and 12.11 at $\bar{d}=10,12$, and 15 , respectively, while $E_{1}^{(G P)} / N=12.10$. This indicates that many-body effects can be recovered only by a full HNC treatment also for a finite number of atoms. However, the solution of the fully correlated HNC Hartree equation, even if feasible in principle, is very cumbersome, computationally time consuming, and numerically delicate. For this reason we have decided to estimate these effects by adopting a local-density-type approach to $V_{\text {corr }}$. We approximate $V_{\text {corr }} \sim V_{\text {corr }}^{L D}$, where

$$
V_{c o r r}^{L D}=\frac{1}{N} \int d \mathbf{r}_{1} \rho_{1}\left(\mathbf{r}_{1}\right) E_{H N C}^{h o m}\left(\rho_{1}\right) .
$$

$E_{H N C}^{h o m}\left(\rho_{1}\right)$ is the HNC homogeneous gas energy per particle at density $\rho_{1}$.

The minimization of the energy gives the HNC correlated Hartree equation $\left(\mathrm{CH}_{H N C}\right)$,

$$
\begin{aligned}
& {\left[-\frac{1}{2} \nabla_{\frac{r}{r}}^{2}+\frac{1}{2} \bar{r}^{2}+E_{1, H N C}^{h o m}\left(x_{l o c}\right)+x_{l o c} \frac{\partial E_{1, H N C}^{h o m}\left(x_{l o c}\right)}{\partial x_{l o c}}\right] \psi_{1}(\bar{r})} \\
& \quad=\mu_{1} \psi_{1}(\bar{r})
\end{aligned}
$$


TABLE I. Chemical potentials $\mu_{1}$, ground-state energies per particle $\mathrm{E}_{1} / \mathrm{N}$, and root mean-square radii $R_{r m s}$, of $N{ }^{87} \mathrm{Rb}$ atoms in an isotropic trap $(\omega / 2 \pi=77.78 \mathrm{~Hz})$ in TF approximation or solving the GP [Eq. (2)], the MGP [Eq. (5)], and the correlated Hartree HNC [Eq. (17)] equations. The $N=1.5 \times 10^{7}$ row refers to the Na case $(\omega / 2 \pi=230 \mathrm{~Hz})$. Energies are in units of $\hbar \omega$ and lengths are in units of $a_{H O}$.

\begin{tabular}{lrrrrrrrrrrr}
\hline \hline \multicolumn{1}{c}{$\mu_{1}$} & \multicolumn{1}{c}{$E_{1} / N$} & & \multicolumn{3}{c}{$R_{r m s}$} \\
\hline$N$ & \multicolumn{1}{c}{ TF } & \multicolumn{1}{c}{ GP } & \multicolumn{1}{c}{ MGP } & HNC & \multicolumn{1}{c}{ TF } & \multicolumn{1}{c}{ GP } & MGP & HNC & GP & MGP & HNC \\
\hline $10^{3}$ & 2.66 & 3.04 & 3.06 & 3.04 & 1.90 & 2.43 & 2.43 & 2.43 & 1.65 & 1.66 & 1.66 \\
$10^{4}$ & 6.67 & 6.87 & 6.92 & 6.89 & 6.87 & 5.04 & 5.08 & 5.04 & 2.44 & 2.45 & 2.44 \\
$10^{5}$ & 16.75 & 16.85 & 17.07 & 16.94 & 11.96 & 12.10 & 12.25 & 12.20 & 3.80 & 3.84 & 3.83 \\
$10^{6}$ & 42.07 & 42.12 & 42.97 & 42.53 & 30.05 & 30.12 & 30.66 & 30.48 & 6.01 & 6.10 & 6.06 \\
$10^{7}$ & 105.68 & 105.70 & 108.75 & 107.20 & 75.49 & 75.52 & 77.48 & 76.85 & 9.52 & 9.74 & 9.64 \\
$1.5 \times 10^{7}$ & 91.07 & 91.10 & 92.41 & 91.67 & 65.05 & 65.09 & 65.92 & 65.66 & 8.84 & 8.92 & 8.90 \\
$10^{8}$ & 265.46 & 265.47 & 275.89 & 273.58 & 189.61 & 189.63 & 196.45 & 194.74 & 15.08 & 15.44 & 15.38 \\
\hline \hline
\end{tabular}

where we have again introduced the scaled unities and the local gas parameter, $x_{l o c}(\bar{r})=\rho_{1}(\bar{r}) a^{3}=N \bar{a}^{3}\left|\psi_{1}(\bar{r})\right|^{2}$.

The calculations have been performed for the ${ }^{87} \mathrm{Rb}$ scattering length. The scaled energies per particle and the root mean-square radii are reported in Table I for particle numbers from $10^{3}$ to $10^{8}$. The table also shows the results obtained by neglecting the kinetic-energy term in the GrossPitaevskii equation. This approach, loosely called the Thomas-Fermi (TF) approximation, has been discussed in the literature and allows for deriving simple analytical expressions [10]. The differences between this Thomas-Fermi approach and a rigorous one have been recently discussed [21,22] for spatially inhomogeneous Bose condensates. Local-density approximation has been used $[1,5]$ to estimate corrections to the Gross-Pitaevskii for the ground and excited states within the Thomas-Fermi approximation and retaining only the first correction in Eq. (1). The second correction is negative and partially cancels the first one. For instance, the cancellations go from $\sim 15 \%$ for $N=10^{4}$ to $\sim 40 \%$ at $N=10^{6}$ if we just take the central densities, whereas the final energy is reduced by $\sim 15 \%$ at $N=10^{6}$ and it is practically unaffected by the second correction at lower $N$ values.

As expected, the Thomas-Fermi results are close to the Gross-Pitaevskii ones when $N$ becomes large. The differ-

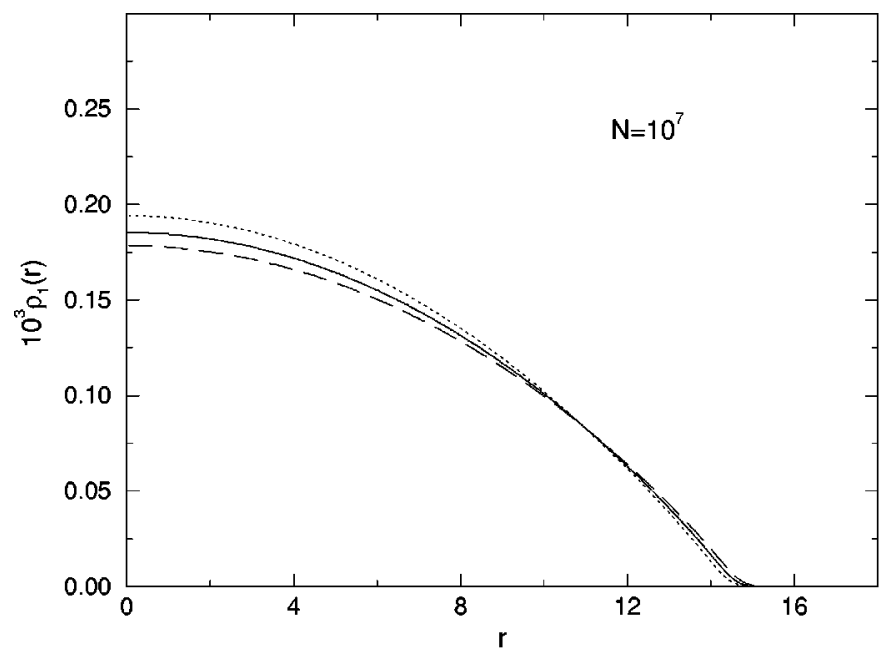

ences between GP and MGP increase with the number of particles and are of the order of $4 \%$ for the chemical potential and $2.5 \%$ for the energy at $N=10^{7}$. The higher-order terms in the low-density expansion always have a repulsive effect. The same behavior is shown by the HNC results, which, however, are less repulsive than MGP at the large $N$ values.

We notice that if one uses the Gross-Pitaevskii solution to perturbatively estimate the MGP energy, then the correction is negative (at $\left.N=10^{7}, \Delta E_{1}=-4.54\right)$. The nonlinear character of Eq. (5) is responsible for this discrepancy.

The density profile (normalized to unity) for $N=10^{7}$ particles is given in Fig. 2. For this large number of particles the TF and GP densities are close, whereas the more repulsive MGP and HNC solutions lower the central density, expanding the density distribution and providing a larger radius, as shown in Table I.

We have also considered a system of $N=1.5 \times 10^{7} \mathrm{Na}$ atoms $(a=27.5 \AA)$ in a spherical trap having a frequency of $230 \mathrm{~Hz}$. These conditions roughly correspond to those of the experiment described in Ref. [4]. The results are shown in the last row of the table and in Fig. 2. The effects of the correlations are similar to those found in the large $N \mathrm{Rb}$ cases. The energy increases by $\sim 1 \%$ and the rms radius by $\sim 0.7 \%$ respect to GP. The HNC central density is slightly reduced.

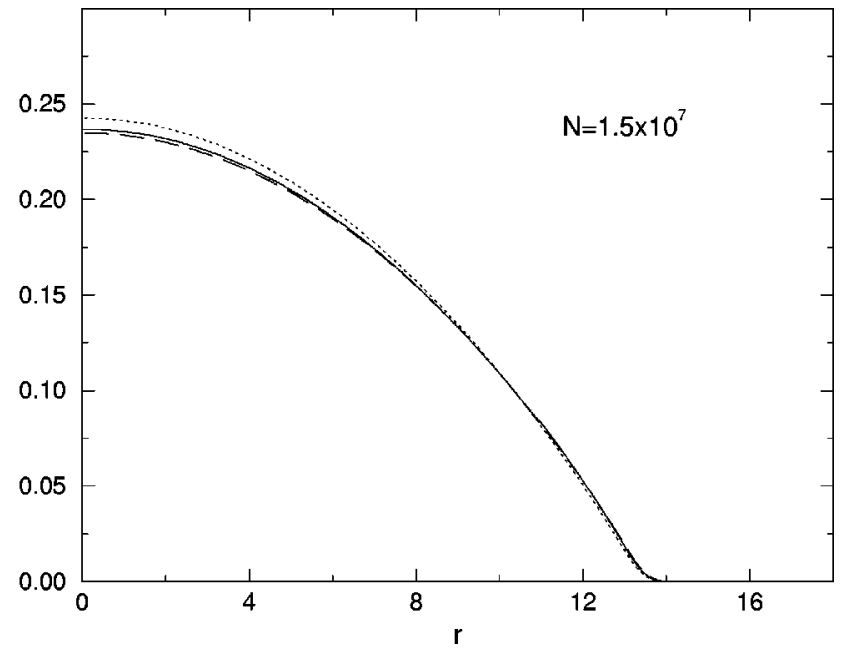

FIG. 2. Density profiles for $N=10^{7} \mathrm{Rb}$ atoms and for $N=1.5 \times 10^{7} \mathrm{Na}$ atoms in different approaches (dotted line, Gross-Pitaevskii; dashed line, modified Gross-Pitaevskii; solid line, hypernetted chain). Densities are normalized to unity and distances are in units of $a_{H O}$. 
In conclusion, we find that both higher-order terms in the low-density expansion (beyond the Gross-Pitaevskii approach and evaluated in local-density approximation) and explicit dynamical correlations (induced by the strong repulsion) have effects of the order of percent in the highest $N$ cases we have studied. In particular, correlations lower the $N=10^{8}$ energy with respect to the mean field by $2.7 \%$ (the effect is larger in the density, lowered by $\sim 6 \%$ ). The average value of the local gas parameter, $x_{l o c}$, is $\left\langle x_{l o c}\right\rangle=2$ $\times 10^{-4}$, and the homogeneous gas correction is $\sim 3.9 \%$ at the same $x$ value. In more extreme conditions (higher $N$ values or stronger trapping potentials) corrections beyond mean field may become even larger and detectable.

In this respect, correlated basis function theory may play a prominent role. In addition, it allows for a fully microscopic investigation for any type of potential. This is particularly interesting in view of its application to atoms having negative scattering lengths, as ${ }^{7} \mathrm{Li}$. In this case the potential exhibits an attractive part and the Gross-Pitaevskii equation has a metastable solution only if $N$ does not exceed some critical value $N_{c}$. A recent study [23], which makes use of an effec- tive interaction, has shown that a new branch of Bose condensate may appear at higher densities. Correlated basis function theory may provide further insights into this problem having access to the full structure of the potential itself.

The introduction of the hypernetted-chain energy functional derived by the homogeneous hard-spheres system provides a quick and probably reliable way of embodying correlation effects into the treatment of the trapped atoms. We see as a particular appeal of this approach the clear possibilty of extending it to nonspherical traps, as in real experiments. Moreover, as already stated, potentials other than the simple hard-sphere one may be easily handled.

\section{ACKNOWLEDGMENTS}

One of us (A.F.) wants to thank Ennio Arimondo, Stefano Fantoni, and Riccardo Mannella for several stimulating discussions. This research was partially supported by DGICYT (Spain) Grant No. PB95-1249, the agreement CICYT (Spain)-INFN (Italy), and the Acción Integrada program (Spain-Italy).
[1] F. Dalfovo, S. Giorgini, L. Pitaevskii, and S. Stringari, Rev. Mod. Phys. 71, 463 (1999).

[2] A.S. Parkins and D.F. Walls, Phys. Rep. 303, 1 (1998).

[3] L.P. Pitaevskii, Zh. Eksp. Teor. Fiz. 40 (1961) [Sov. Phys. JETP 13, 451 (1961)]; E.P. Gross, Nuovo Cimento 20, 454 (1961).

[4] D.M. Stamper-Kurn et al., Phys. Rev. Lett. 81, 500 (1998).

[5] L. Pitaevskii and S. Stringari, Phys. Rev. Lett. 81, 4541 (1998).

[6] K. Ziegler and A. Shukla, Phys. Rev. A 56, 1438 (1997); 57, 1464(E) (1998).

[7] W. Krauth, Phys. Rev. Lett. 77, 3695 (1996).

[8] M. Holzmann et al., Phys. Rev. A 59, 2956 (1999).

[9] A.L. Fetter and J.D. Walecka, Quantum Theory of ManyParticle Sytems (McGraw-Hill, New York, 1971).

[10] M. Edwards and K. Burnett, Phys. Rev. A 51, 1382 (1995).

[11] F. Dalfovo and S. Stringari, Phys. Rev. A 53, 2477 (1996).

[12] P. Capuzzi and E.S. Hernández, Phys. Rev. A (to be published).

[13] E. Cerboneschi, R. Mannella, E. Arimondo, and L. Salasnich,
Phys. Lett. A 249, 495 (1998).

[14] S. Fantoni and A. Fabrocini, in Microscopic Quantum ManyBody Theories and Their Applications, edited by J. Navarro and A. Polls, Lecture Notes in Physics Vol. 510 (SpringerVerlag, Berlin, 1998), p. 119.

[15] A. Fabrocini, S. Fantoni, A. Polls, and S. Rosati, Nuovo Cimento A 56, 33 (1980).

[16] R. Jastrow, Phys. Rev. 98, 1479 (1955).

[17] E. Krotscheck, Nucl. Phys. A 465, 461 (1987).

[18] V.R. Pandharipande and K.E. Schmidt, Phys. Rev. A 15, 2486 (1977).

[19] J. Boronat (private communication).

[20] K.T.R. Davies, H. Flocard, S. Krieger, and M.S. Weiss, Nucl. Phys. A 342, 111 (1980).

[21] E. Timmermans, P. Tommasini, and K. Huang, Phys. Rev. A 55, 3645 (1997).

[22] P. Schuck and X. Vinas (private communication).

[23] A. Parola, L. Salasnich, and L. Reatto, Phys. Rev. A 57, R3180 (1998). 